\title{
Possible involvement of MIP-1 $\alpha$ in the recruitment of osteoclast progenitors to the distal tibia in rats with adjuvant-induced arthritis
}

\author{
Kazuko Toh ${ }^{1,2}$, Toshio Kukita ${ }^{1}$, Zhou Wu ${ }^{2}$, Akiko Kukita ${ }^{3}$, Ferry Sandra ${ }^{1}$, Quan Yong Tang ${ }^{1}$, \\ Hisayuki Nomiyama ${ }^{4}$ and Tadahiko Iijima ${ }^{2}$ \\ ${ }^{1}$ Department of Oral Cellular and Molecular Biology, Division of Oral Biological Science; ${ }^{2}$ Department of Oral \\ Anatomy and Cell Biology, Faculty of Dental Sciences, Kyushu University, Fukuoka, Japan; ${ }^{3}$ Department of \\ Microbiology, Saga Medical School, Saga, Japan and ${ }^{4}$ Department of Biochemistry, Faculty of Medicine, \\ Kumamoto University, Kumamoto, Japan
}

\begin{abstract}
In the rat model of rheumatoid arthritis, a marked formation of osteoclasts is found in the distal tibia and the metatarsal bone. It was therefore postulated that osteoclast progenitors would be increased in the bone marrow cavities of rats with adjuvant-induced arthritis (AA rats). Bone marrow cells obtained from tibia of AA rats were cultured to form cells in the osteoclast lineage to access the number of osteoclast progenitors. Unexpectedly, only a suppressed level of osteoclast progenitors was detected in the diaphyseal bone marrow of tibia in AA rats. Distribution of osteoclast progenitors in the bone marrow cavity was examined, and it was shown that osteoclast progenitors accumulated in the distal tibia. Macrophage inflammatory protein (MIP)-1 $\alpha$, an osteoclastogenic CC chemokine, was expressed in ED-1-positive macrophages localizing in the distal tibia with marked bone destruction. Chemotaxis studies showed that MIP- $1 \alpha$ expressed significant activity towards bone marrow cells. The suppressed level of osteoclastogenesis in bone marrow cells of AA rats was restored to a normal level by the addition of MIP-1 $\alpha$. It was suggested that MIP- $1 \alpha$ is involved in the migration of osteoclast progenitors to the distal tibia as well as in osteoclastogenesis in AA rats. In these rats, in situ hybridization of the distal tibia with a high level of bone destruction showed significant expression of Receptor activator nuclear factor $\kappa \mathrm{B}$ ligand (RANKL) messenger RNA in aggregates of multinucleated osteoclast-like cells present in the bone marrow cavity, a unique pathological feature for these rats. Migrated osteoclast progenitors are thought to be efficiently differentiated into osteoclasts in response to RANKL expressed by the aggregates of osteoclastlike cells under the influence of the MIP-1 $\alpha$. Such positive-feedback regulation of osteoclastogenesis could result in the highest recruitment of active osteoclasts in the area of marked bone destruction.
\end{abstract}

Laboratory Investigation (2004) 84, 1092-1102, advance online publication, 14 June 2004; doi:10.1038/labinvest.3700132

Keywords: adjuvant arthritis; bone marrow; osteoclastogenesis; migration of progenitors; distal tibia; MIP-1 $\alpha$; RANKL; chemotaxis

Rats with adjuvant-induced arthritis (AA rats) have been frequently utilized as an experimental model of human rheumatoid arthritis (RA). These rats develop polyarthritis after a single intradermal injection with complete Freund's adjuvant. The resulting severe arthritis is characterized by the

Correspondence: Dr T Kukita, PhD, Department of Oral Cellular and Molecular Biology, Division of Oral Biological Science, Faculty of Dental Sciences, Kyushu University, 3-1-1 Maidashi, Fukuoka 812-8582, Japan.

E-mail: kukitat@dent.kyushu-u.ac.jp

Received 10 December 2003; revised 6 April 2004; accepted 13 April 2004; published online 14 June 2004 infiltration of mononuclear cells into synovial tissues, pannus formation, and articular cartilage erosion in the ankle joints. $^{1,2}$ Extensive bone resorption is accompanied with arthritis in the distal diaphysis of the tibia as well as in the tarsal bone. Osteoclastogenesis is markedly stimulated at sites of bone destruction, and significant aggregates of osteoclast-like multinucleated cells are frequently observed in the bone marrow cavity of areas of severe bone destruction. ${ }^{3}$ Bone erosion causes distal diaphysis of tibia and tarsal bones located near ankle joints with severe arthritis, suggesting that infiltration of inflammatory cells into adjacent bone tissues triggers extensive osteoclastogenesis. ${ }^{4}$ How- 
ever, only limited information has so far been available concerning the recruitment of abundant osteoclasts in the site of severe bone destruction. Osteoclastogenesis occurs in the bone marrow cavity from the hematopoietic stem cells present in the bone marrow. In AA rats, marked osteoclastogenesis is observed in the marrow space of distal tibia and tarsal bone. It is reasonable to think that adjuvant injection might modulate the number and the localization of osteoclast progenitors present in the bone marrow cavity.

Osteoclasts are multinucleated giant cells formed by the fusion of mononuclear precursors derived from hematopoietic stem cells. In vitro studies have demonstrated an involvement of various inflammatory cytokines such as tumor necrosis factor- $\alpha$ (TNF$\alpha$ ), interleukin-1 $\alpha$ (IL-1 $\alpha$ ), and interleukin-6 (IL-6), in the regulation of osteoclastogenesis. ${ }^{5}$ Excessive osteoclastogenesis induced by these cytokines often triggers pathological bone destruction at particular bone sites. ${ }^{4}$ Receptor activator nuclear factor $\kappa \mathrm{B}$ ligand (RANKL), believed to be the most important cytokine for normal osteoclastogenesis, is also involved in inflammatory bone resorption. ${ }^{6}$ In addition to cytokines being involved in osteoclastogenesis, an involvement of factors bearing the ability of chemotaxis against cells in the osteoclast lineage should be considered.

Macrophage inflammatory protein (MIP)- $1 \alpha^{7,8}$ is a CC chemokine and has chemotactic activity on monocytes, lymphocytes, dendritic cells, eosinophils, and basophils. This chemokine also acts as a chemotactic factor for mature osteoclasts ${ }^{9}$ as well as for osteoclast precursors. ${ }^{10}$ Previously, we demonstrated that MIP- $1 \alpha$ induces osteoclastogenesis in a rat bone marrow culture system. ${ }^{11,12}$ Choi et $a l^{13}$ and Han et $a 1^{14}$ showed that MIP-1 $\alpha$ is involved in the pathological osteoclastogenesis associated with multiple myeloma.

In this paper, we focus on how bone marrow cells are modulated as the source of osteoclasts during adjuvant-induced arthritis. We also examine the expression of MIP- $1 \alpha$ and RANKL in the area of severe bone destruction of rats with arthritis. We have detected a migration of osteoclast progenitors toward the distal epiphysis of tibia, in which MIP- $1 \alpha$ is involved as a chemotactic as well as an osteoclastogenic factor. We also detected a significant expression of RANKL in the aggregates of osteoclastlike multinucleated giant cells, a unique histological feature of AA rats.

\section{Materials and methods}

\section{Materials}

Lewis rats were obtained from SEAC Yoshitomi (Fukuoka, Japan). Culture vessels were from Becton Dickinson Labware (Lincoln Park, NJ, USA) and minimum essential medium alpha medium $(\alpha-M E M)$ was purchased from GIBCO BRL (Grand
Island, NY, USA) and 1 1 2,25 -dihydroxyvitamin $\mathrm{D}_{3}$ $\left(1 \alpha, 25(\mathrm{OH})_{2} \mathrm{D}_{3}\right)$ was from BIOMOL Research Laboratories Inc. (Plymouth Meeting, PA, USA). Fetal bovine serum (FBS) was from Bio Whittaker (Walkersville, MD, USA). The tartrate-resistant acid phosphatase (TRAP) staining kit was from SigmaAldrich (St Louis, MO, USA). RANKL, TNF- $\alpha$, MIP- $1 \alpha$, and anti-rat MIP- $1 \alpha$ were obtained from Peprotec Science (London, England). RANKL cDNA was kindly provided by Dr Nishimura of Osaka University Graduate School of Dentistry.

\section{Animal Treatment}

All animal treatments were performed according to the guidelines for the care and use of laboratory animals at Kyushu University.

\section{Induction of Adjuvant Arthritis}

Adjuvant arthritis was induced by a single intradermal injection of $25 \mathrm{mg} / \mathrm{kg}$ heat-killed Mycobacterium butyricum (Difco Laboratories, Detroit, MI, USA) suspended in mineral oil at the tail base of female Lewis rats (Seac Yoshitomi, Fukuoka, Japan), weighing 100-110 g, under deep ether anesthesia. For the control experiments, rats were injected with mineral oil alone. The rats were housed for $24 \mathrm{~h}$ in a $12-\mathrm{h}$ light/dark cycle with free access to water and chow.

\section{Bone Marrow Cultures for Forming Osteoclast-Like MNCs and Bone Resorption Assay}

Rat bone marrow cells were obtained from tibia of AA rats (17 days after adjuvant injection) and control rats. After cutting off both ends (proximal and distal tibia), bone marrow cells were flushed out with serum-free $\alpha$-MEM using a syringe attaching 25-gauge needle. Marrow cells were also flushed out from each end of the tibia. The cells were cultured in 24 -multiwell culture plates $\left(500 \mu \mathrm{l}\right.$ of $2 \times 10^{6}$ cells/ $\mathrm{ml}$ per well) for forming osteoclast-like multinucleated cells in $\alpha$-MEM containing $15 \%$ FBS in the presence of $10^{-8} \mathrm{M} 1 \alpha, 25(\mathrm{OH})_{2} \mathrm{D}_{3}$ and $10 \%(\mathrm{v} / \mathrm{v})$ heat-treated conditioned medium derived from the rat osteoblastic cell line ROS 17/2.8 (ht ROSCM), as described previously. ${ }^{15}$ After culturing for 4 days in the presence of various concentrations of TNF- $\alpha$ and RANKL, the cells were stained for TRAP using an acid phosphatase staining kit (Sigma, St Louis, MO, USA). For estimating function of osteoclast-like MNCs, cells were detached from the plastic culture wells using $0.02 \%$ EDTA in PBS and replated on dentine slices (4 $\mathrm{mm}$ in diameter) followed by culture for 3 days. Resorption pits were stained with $1 \%$ of toluidine blue after removing the cells by ultrasonification in $0.25 \mathrm{M}$ ammonium hydroxide. 


\section{Bone Marrow Cultures for Forming Mononuclear Preosteoclast-Like Cells}

Bone marrow cells were obtained from the tibia of AA rats (17 days after adjuvant injection). Bone marrow stromal cells were depleted using a Sephadex G10 column as described previously. ${ }^{16}$ These cells were cultured in 24-multiwell culture plates $\left(1 \times 10^{6}\right.$ cells/well $)$ for 4 days in $\alpha$-MEM containing $15 \%$ FBS and $10^{-8} \mathrm{M} 1 \alpha, 25(\mathrm{OH})_{2} \mathrm{D}_{3}$ and $10 \%(\mathrm{v} / \mathrm{v}) \mathrm{ht}$ ROSCM. The absence of stromal cells was confirmed in the Sephadex G10-passed bone marrow cells after culture in $100 \mathrm{~mm}$ dishes for 2 weeks.

\section{Chemotaxis Assays}

Chemotaxis assays were performed using a transwell chamber according to the manufacturer's protocol (pore size of $8 \mu \mathrm{m}$, Becton Dickinson Labware, NJ, USA). Cells were allowed to migrate for $4 \mathrm{~h}$ at $37^{\circ} \mathrm{C}$ followed by fixation in $4 \%$ formaldehyde and staining with hematoxylin and eosin. The number of cells that migrated to the lower side of the filter was determined by microscopy.

\section{Immunohistochemistry}

Tissue blocks were taken from the hind paws (tarsal bones and tibia) and were then further immersed in the same fixative for $12 \mathrm{~h}$ at $4^{\circ} \mathrm{C}$, washed in PBS overnight at $4^{\circ} \mathrm{C}$. These tissues blocks were decalcified in $10 \%$ EDTA for 3 weeks at $4^{\circ} \mathrm{C}$. After decalcification, the tissue blocks were washed overnight in PBS containing 30\% sucrose, embedded in OCT compounds and frozen in dry ice/isopentane. Sections $(10 \mu \mathrm{m}$ thick) of ankle joints including tibia-tarsal-calcaneus bones were prepared using Frigocut 2800N, Leica (Germany). Immunohistochemistry was performed as described previously. $^{2}$ Briefly, sections were incubated with rabbit polyclonal anti-rat MIP- $1 \alpha$ antibody (1:100 dilution; PEPROTECH EC LTD, London, UK) in a humidified chamber overnight at $4^{\circ} \mathrm{C}$ after blocking nonspecific binding with $10 \%$ donkey serum for $30 \mathrm{~min}$ at room temperature. After washing with PBS, sections were incubated with biotinylateddonkey anti-rabbit IgG (1:200 dilution; Jackson Immunoresearch Laboratories, West Grove, PA, USA) for $1 \mathrm{~h}$ at room temperature followed by being reacted with peroxidase-conjugated streptavidinbiotin complex (1:300 dilution; Dako Japan, Kyoto, Japan) for $30 \mathrm{~min}$ at room temperature. Color development was performed using a DAB substrate kit (Vector Laboratories, Burlingame, CA, USA).

\section{Double-Immunofluorescence Staining}

Tissue sections were hydrated and pretreated with $10 \%$ normal donkey serum for $30 \mathrm{~min}$ at room temperature. Sections were incubated with primary antibodies, which are rabbit polyclonal anti-rat MIP$1 \alpha$ antibody (1:100 final dilution, PEPROTECH EC LTD, London, UK) mixed with mouse monoclonal anti-macrophage antibody ED-1 (1:200 final dilution, BMA Biomedicals, Augst, Switzerland) in a humidified chamber overnight at $4^{\circ} \mathrm{C}$. After washing with PBS, the sections were incubated with a mixture of fluorescence isothiocyanate (FITC)-conjugated donkey anti-rabbit IgG (1:200 final dilution) and rhodamine-conjugated donkey anti-mouse IgG (1:500) for $1 \mathrm{~h}$ at RT. After washing with PBS, the sections were observed under fluorescence microscopy (Olympus, AX70, Japan).

\section{Preparation of RNA Probes}

The BamHI-EcoRI fragment excised from the pSecTag213-RANKL encoding murine RANKL cDNA was subcloned into the BamHI-EcoRI site of pBluescript KS (+). After amplifying in Escherichia coli, plasmid DNA was purified and linearized by digestion with BamHI or EcoRI. RNA probes were prepared with an RNA synthesis kit (Nippongene, Toyama, Japan). The EcoRI-linearized plasmid DNA was utilized as the DNA template for preparing the antisense RNA probe using T7 RNA polymerase. The BamHI-linearized plasmid DNA was used as the template DNA for preparing the sense RNA probe using T3 RNA polymerase.

\section{In Situ Hybridization}

AA rats were anesthetized with Nembutal (Abbot Laboratories, North Chicago, IL, USA) and perfused with $4 \%$ paraformaldehyde in PBS ( $\mathrm{pH} \mathrm{7.4).} \mathrm{Tissue}$ blocks were taken from the hind paws (tarsal bones and tibia) and immersed in the same fixative for $6 \mathrm{~h}$ at $4^{\circ} \mathrm{C}$, washed in PBS, and demineralized at $4^{\circ} \mathrm{C}$ for 3 weeks in Morse's solution $(22.5 \%$ formic acid, $10 \%$ sodium citrate). The tissue blocks were dehydrated through a graded series of ethanol followed by being embedded in paraffin. Serial sections of $5 \mu \mathrm{m}$ thickness were cut and transferred onto silane-coated glass slides. After dewaxing, in situ hybridization was performed as described previously. ${ }^{12,17}$

\section{Statistical Analysis}

All data obtained from cell cultures were statistically analyzed by Student's $t$-test.

\section{Results}

\section{Histological Observations of Distal Diaphysis of the Tibia in Adjuvant Arthritis}

Clinical symptoms of AA rats were observed 10 days after adjuvant injection, and increased during the 
next 3 weeks with a higher level of redness and swelling of each hind paw. As controls, rats were injected with mineral oil alone and exhibited no histological abnormality (Figure 1a). Extensive bone destruction was observed in the distal epiphysis of tibia and in the tarsal bone in rats with arthritis at 4 weeks after the adjuvant injection (Figure 1b). Aggregates of numerous multinucleated osteoclastlike cells were observed in the bone marrow cavity of distal tibia and tarsal bones in AA rats (Figure 1c).

\section{Modulation of Osteoclastogenic Activity in Bone Marrow of AA Rats}

Osteoclast differentiation is markedly increased in AA rats. We postulated that the potential of bone marrow cells to form osteoclasts was augmented in AA rats. Bone marrow cells obtained from the diaphyseal part of the tibia of control and AA rats (17 days after adjuvant injection) were cultured to form osteoclast-like cells and compared with normal rats. A significant induction of osteoclastogenesis was observed in response to RANKL and TNF- $\alpha$ in bone marrow cells obtained from normal rats. However, unexpectedly, only a suppressed level of osteoclastogenesis was observed in bone marrow cells obtained from AA rats, even if marrow cells were stimulated by high doses of RANKL and TNF- $\alpha$ (Figure 2a). Consistent results were also seen in preosteoclast formation study (Figure 2b). These results suggested that the number of osteoclast progenitors was markedly decreased in bone marrow cavity of diaphyseal part of the tibia in AA rats.

\section{Modulated Distribution of Osteoclast Progenitors in the Bone Marrow Cavity in AA Rats}

To determine the distribution of osteoclast progenitors in the bone marrow cavity of AA rats, we compared the ability of bone marrow cells to form osteoclasts in different areas of the marrow cavity of the tibia. Figure 3 compares potential osteoclastogenesis among three parts of the tibia. In diaphysis, a significant suppression of osteoclastogenesis was observed in AA rats with marked osteoclastogenesis in the distal part of the tibia (also shown in Table 1). It is suggested that osteoclast progenitors migrated from diaphysis and accumulated in the distal part of the tibia in AA rats.

\section{MIP-1 $\alpha$-Induced Osteoclastogenesis in Bone Marrow Cells of AA Rats}

To address why osteoclastogenesis was severely suppressed in the bone marrow cells obtained from diaphysis of the tibia (Figure 2) in AA rats, we postulated that other cytokines might have osteoclastogenic or chemotactic activity against cells in osteoclast lineage. MIP- $1 \alpha$ is known to have osteoclastogenic as well as chemotactic activity against cells in the osteoclast lineage.$^{9,11}$ As shown in Figure 4, MIP-1 $\alpha$ significantly stimulated osteoclastogenesis dose-dependently from bone marrow cells of AA rats and reached a level similar to normal control rats at $10 \mathrm{ng} / \mathrm{ml}$. A significant increase in the number of resorption pits was observed when MIP-1 $\alpha$-treated osteoclast-like MNCs (formed from
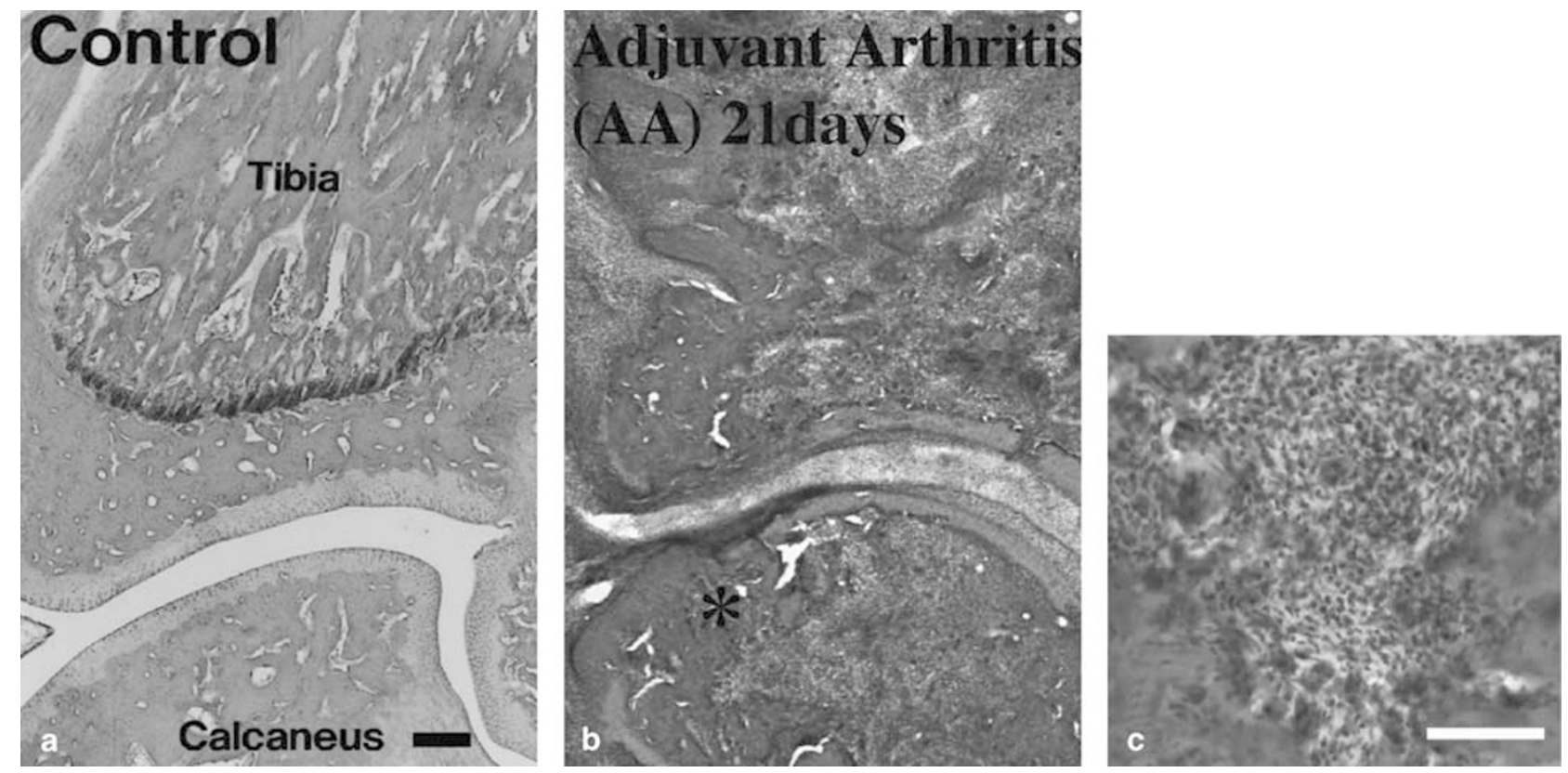

Figure 1 Histological observation of distal diaphysis of the tibia in AA rats. (a) Control rats: Rats injected with mineral oil alone exhibited no clinical symptoms of AA. (b) AA rats: Extensive bone destruction was observed in distal epiphysis of the tibia as well as in the tarsal bone. (c) A high magnification view of (b) ${ }^{*}$ : A large number of osteoclasts are observed in the bone marrow cavity. (a, b) Bar $250 \mu \mathrm{m}$, (c) Bar $100 \mu \mathrm{m}$. 
1096
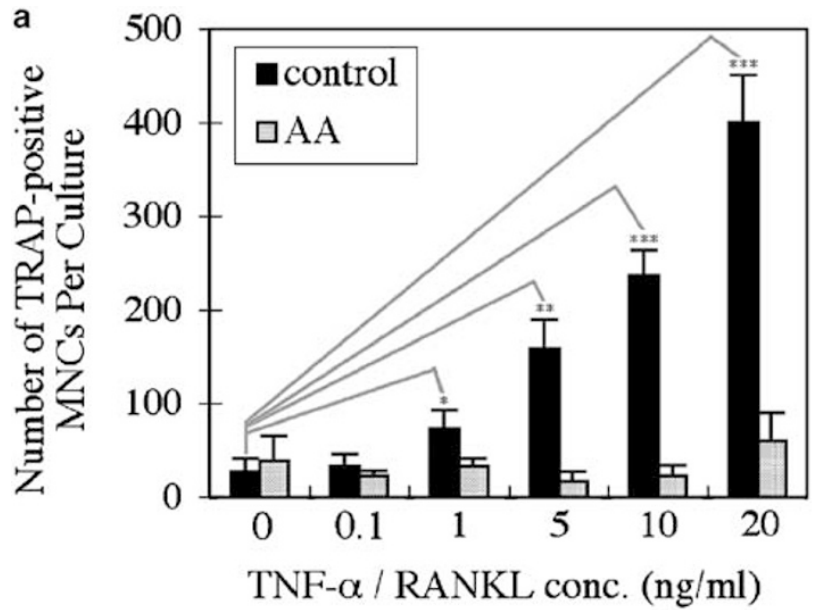

b
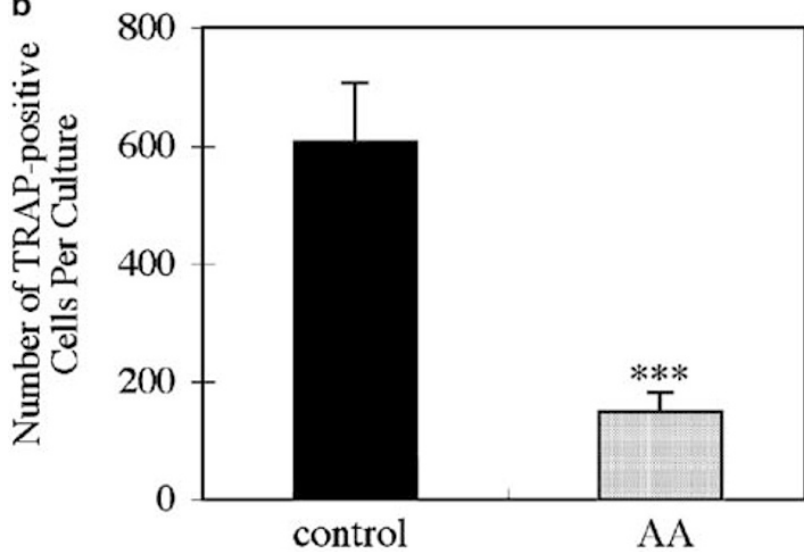

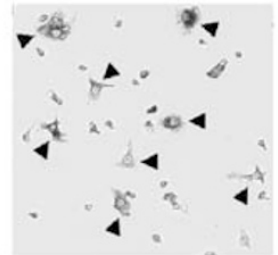

control

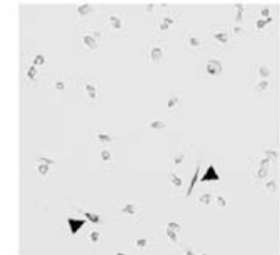

AA
Figure 2 Significant suppression of osteoclastogenesis in diaphyseal bone marrow cells from AA rats. (a) Whole bone marrow culture form osteoclast-like multinucleated cells (MNCs): Bone marrow cells were obtained and cultured to form osteoclast-like MNCs from normal (control) or AA rats as described in Materials and methods. At 4 days of culture, cells were stained for TRAP. While significant osteoclastogenesis was observed in normal bone marrow cultures in response to RANKL and TNF- $\alpha$, marked suppression of osteoclastogenesis was observed in bone marrow cultures of AA rats. The mean number of nuclei per MNC was not significantly altered by stimulation with these factors. Data represent the mean number of TRAP-positive MNCs \pm s.e.m. in quadruplicate cultures. (b) Stromal cell-deprived bone marrow culture form preosteoclasts: Rat bone marrow cells deprived of stromal cells were cultured to form preosteoclast in 24-multiwell culture plates as described in Materials and methods. Cells were stained for TRAP and the number of TRAP-positive mononuclear cells were counted (upper panel). Data represent mean \pm s.e.m. from quadruplicate cultures. Marked suppression of preosteoclast formation is apparent in culture of bone marrow cells from AA rats (lower panel). Data were analyzed by Student's $t$-test. ${ }^{*} P<0.05,{ }^{* *} P<0.01,{ }^{* * *} P<0.001$. Data represent a typical experiment from three independent experiments. Arrowheads: TRAP-positive preosteoclast-like cells.

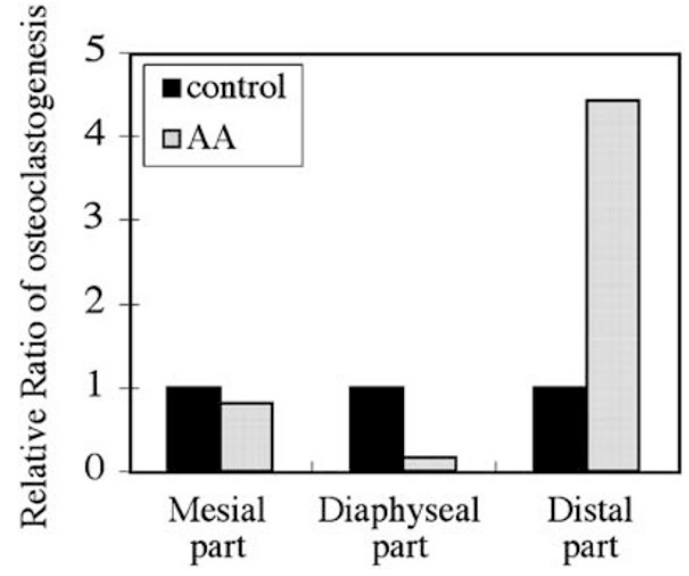

Figure 3 Distribution of osteoclast progenitors in the bone marrow cavity. Comparison of the potential ability of osteoclastogenesis among different portions (mesial parts, diaphyseal parts, distal parts) of the tibia. Rat bone marrow cells obtained from each part of the tibia were cultured in 24-multiwell culture plates in the presence of $10^{-8} \mathrm{M} 1 \alpha, 25-(\mathrm{OH})_{2} \mathrm{D}_{3}$ and ht ROSCM for 4 days as described in Materials and methods. These culture cells were stained for TRAP. Data represent relative ratios of osteoclastogenesis as shown in the following formula:

\section{Relative ratio of osteoclastogenesis $=$ (number of TRAP-positive osteoclast-like MNCs formed from the marrow of AA rats)/(number of TRAP-positive osteoclast-like MNCs formed from normal marrow $) \times 100(\%)$}

Marked suppression of osteoclastogenesis is observed in the diaphyseal part of the tibia; in contrast, significant increase in the osteoclastogenesis is apparent in the distal part of tibia. Data represent a typical experiment from three independent experiments.

Table 1 Distribution of osteoclast progenitors in bone marrow cavity

\begin{tabular}{lcc}
\hline & \multicolumn{2}{c}{ Number of TRAP-position MNC } \\
\cline { 2 - 3 } & Diaphyseal part & Distal part \\
\hline Control rat & $91.8 \pm 28.9$ & $6.7 \pm 5.1$ \\
AA rat & $14.2 \pm 11.6^{*}$ & $31.0 \pm 14.0^{*}$
\end{tabular}

The results are expressed in terms of mean \pm s.d. Rat bone marrow cells were obtained from each part of the tibia and cultured for forming osteoclast-like MNCs as described in the figure legend of Figure 3. Data represent the mean number of TRAP-positive MNCs \pm s.d. from quadruplicate cultures. Numbers of formed MNCs were compared between control rats and AA rats. Data were analyzed by Student's $t$-test. ${ }^{*} P<0.05$. Significant stimulation of osteoclastogenesis was apparent in the distal part of the tibia of AA rats, while marked suppression of osteoclastogenesis was observed in the diaphyseal part of tibia of AA rats. The mean number of nuclei per MNC was not significantly different in each culture.

marrow cells of 17 days of AA rats) were replated on dentin slices (Table 2). These data strongly suggest that osteoclastogenesis is regulated by the presence of MIP- $1 \alpha$ in AA rats. We examined whether other chemokines have similar effects. However, less stimulation of osteoclastogenesis was observed by 


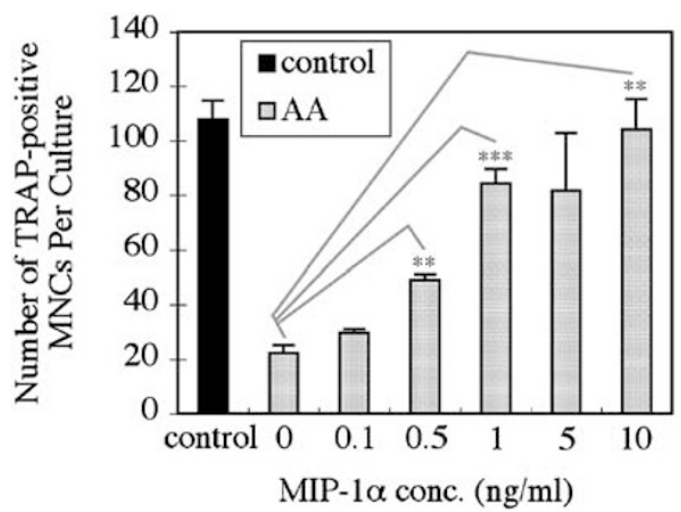

Figure 4 Marked stimulation of osteoclastogenesis by MIP- $1 \alpha$ in bone marrow cells of AA rats. Bone marrow cells obtained from normal rats (control) or AA rats were cultured in the presence of $1 \alpha, 25-(\mathrm{OH})_{2} \mathrm{D}_{3}$ and ht ROSCM as described in Materials and methods. MIP- $1 \alpha$ stimulated osteoclastogenesis from bone marrow cells of AA rats in a dose-dependent manner. Mean number of nuclei per MNC was not significantly changed when cells were treated with MIP-1 $\alpha$. Data represent mean \pm s.e.m. from quadruplicate cultures. Data were analyzed by Student's $t$-test. ${ }^{*} P<0.01,{ }^{* *} P<0.001$. Data represent a typical experiment from three independent experiments.

Table 2 MIP-1 $\alpha$-induced osteoclast-like MNCs efficiently resorb dentin

Conc. of $M I P-1 \alpha$ Number of pits per dentin slice \pm s.d.

\begin{tabular}{rl}
\hline 0 & $26.5 \pm 17.6$ \\
1 & $53.8 \pm 32.7$ \\
10 & $99.8 \pm 45.2 *$
\end{tabular}

The results are expressed in terms of mean \pm s.d. Bone marrow cells obtained from tibia of AA rats (17 days after adjuvant injection) were cultured for forming osteoclast-like MNCs in the presence of various concentrations of MIP- $1 \alpha$. After 3 days of culture, cells were replated on dentin slices ( $4 \mathrm{~mm}$ in diameter) placed in wells of 96-multiwell culture plates and cultured for further 3 days in the absence of any factors followed by assessing the dentin resorption activity of these cells as described in Materials and methods. Data represent mean \pm s.d. from six independent cultures. MIP- $1 \alpha$-treatment $(10 \mathrm{ng} /$ $\mathrm{ml}$ ) significantly increased the number of resorption pits. Data were analyzed by Student's $t$-test. ${ }^{*} P<0.05$. Data represent a typical experiment from three independent experiments.

other chemokines (MIP-1 $\beta$, MIP- $1 \gamma$, RANTES, MCP1, EOTAXIN) (data not shown). These results strongly suggest that MIP- $1 \alpha$ has a unique role in the induction of osteoclastogenesis in AA rats.

\section{Immunohistochemical Localization of MIP-1 $\alpha$ in the Bone Marrow Cavity of Distal Tibia in AA Rats}

We examined whether MIP- $1 \alpha$ is actually expressed in the bone marrow cavity in AA rats. Figure 5 shows the immunohistochemical analysis of the tibia obtained from rats after 21 days of adjuvant injection. While few MIP-1 $\alpha$-positive cells were detected in tibia of normal rats (Figure 5a), numer- ous MIP- $1 \alpha$-positive cells were observed in the distal portion of the tibia in AA rats (Figure $5 b, c$ ). High magnification of the MIP- $1 \alpha$-positive mononuclear cells (Figure 5c) suggested that these cells were likely to be macrophages. To confirm that MIP- $1 \alpha$ expressing cells were macrophages, we performed double immunofluorescence staining using anti-ED1 antigen antibody, a marker of rat macrophages. As shown in Figure 6, most of the ED-1 antigen-positive mononuclear cells expressed MIP-1 $\alpha$. These data strongly suggest that MIP- $1 \alpha$ is produced by macrophages localized in the distal part of the tibia in AA rats.

\section{Ability of MIP-1 $\alpha$ to Induce Migration of Osteoclast Progenitors}

Accumulation of osteoclast progenitors (Figures 2 and 3 ) and localization of MIP- $1 \alpha$ secreting cells (Figures 5 and 6) in the distal part of the tibia in AA rats strongly suggest that osteoclast progenitors present at the diaphyseal part of the tibia migrated to the marrow cavity toward the distal end of the tibia. To obtain evidence for this hypothesis, we examined the chemotactic activity of MIP- $1 \alpha$ toward bone marrow cells obtained from normal rats. Figure 7 shows chemotactic activity of MIP- $1 \alpha$ toward normal rat marrow cells in a chemotaxis chamber. Peak stimulation was obtained at $0.1 \mathrm{ng} / \mathrm{ml}$ of MIP$1 \alpha$. These data clearly demonstrate the chemotactic role of MIP- $1 \alpha$ for bone marrow cells involving osteoclast progenitors.

\section{Expression of RANKL Messenger RNA in Aggregates of Osteoclast-Like Cells, a Unique Pathological} Feature for Rats with Arthritis

RANKL, the most important cytokine for normal osteoclastogenesis, is also a mediator of inflammatory bone resorption. In AA rats, osteoclastogenesis is markedly stimulated at sites of bone destruction. A significant aggregate of osteoclast-like multinucleated cells was frequently observed in the bone marrow cavity in severe bone destruction areas. Figure 8 illustrates expression of RANKL messenger RNA (mRNA) in aggregates of osteoclast-like cells seen in the marrow cavity of AA rats. These data suggest some cooperative role of RANKL with MIP$1 \alpha$ in osteoclast recruitment in the bone marrow cavity of AA rats.

\section{Discussion}

In AA rats, bone tissue is quite abundant in activated osteoclasts, those adhering to the surface of bone, as well as resting osteoclasts localized in large aggregates of giant cells characteristic of AA rats. One possible explanation for such a dramatic up-regulation in osteoclastogenesis is that osteoclast 

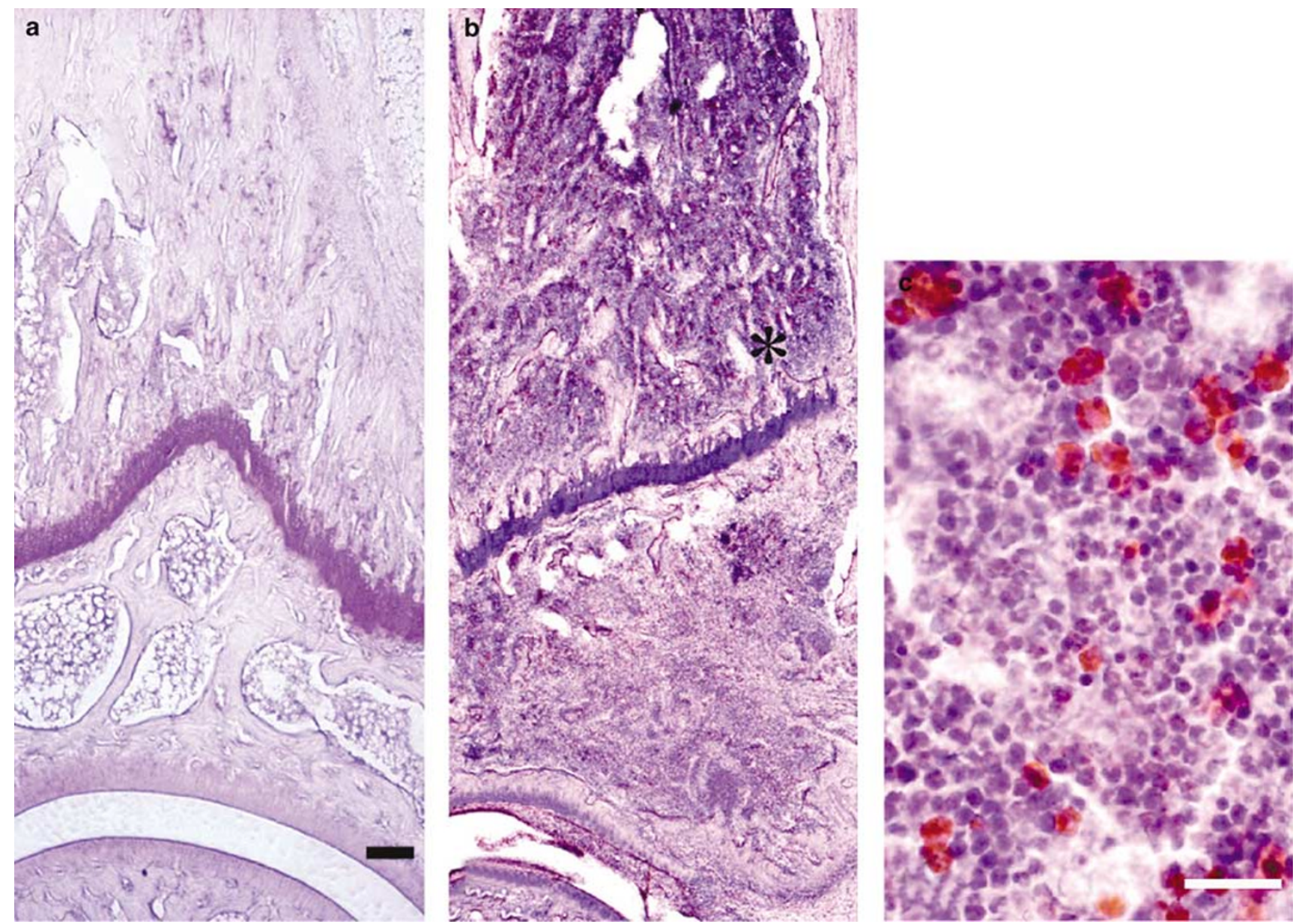

Figure 5 Immunohistochemical localization of MIP-1 $\alpha$-producing cells in the bone marrow cavity of distal tibia in AA rat. (a) Few MIP$1 \alpha$-positive cells were detected in the tibia of normal rats. (b) AA rats 21 days after adjuvant injection: Numerous MIP-1 $\alpha$-positive cells in bone marrow cavity at the distal end of tibia in AA rats after 21 days of adjuvant injection. (c) High magnification view of (b)* (a, b) Bar $125 \mu \mathrm{m}$; (c) Bar $30 \mu \mathrm{m}$.

progenitors increase in the bone marrow cavity of AA rats. We first examined this possibility but obtained disparate results, in which the number of osteoclast progenitors strikingly decreased in the bone marrow cells obtained from the diaphysis of tibia in AA rats. Then, we compared the potency of marrow cells for osteoclastogenesis among bone marrow cells obtained from different parts of the tibia. In AA rats, a significant increase in osteoclastogenic activity was observed in the distal portion of the tibia, compared to that of the normal tibia. Osteoclastogenic activity of the diaphyseal bone marrow was markedly suppressed in AA rats. Our finding shows that osteoclast progenitors in diaphyseal tibia decreased while those in the distal tibia increased during AA. It is highly probable that osteoclast progenitors present in the marrow cavity of tibia have migrated toward distal tibia during the early phase of adjuvant arthritis, probably forming active osteoclasts, which cause severe bone destruction. In normal rat bone marrow cells, a marked stimulation of osteoclastogenesis is observed when both TNF- $\alpha$ and RANKL were added to bone marrow cultures. In contrast, a significant decrease in sensitivity against these factors was observed in diaphyseal marrow cells of AA rats. Suppressed osteoclastogenesis from diaphyseal marrow cells was recovered by the addition of MIP- $1 \alpha$, one of the chemotactic factors for monocytes/macrophages. Bone marrow cells of AA rats are likely to be upregulated in the sensitivity against MIP- $1 \alpha$, while sensitivity to TNF- $\alpha$ and RANKL decreases. Thus, it is suggested that migration of osteoclast progenitors present in the diaphyseal part of the tibia occurs toward the end of the tibia in the early stage of AA (Figure 9). Both a quantitative change, a decrease in the number of osteoclast progenitors by migration of these cells toward distal tibia from the diaphyseal portion, and a qualitative change, modulation of the sensitivity to osteoclastogenic factors, take place. Although MIP-1 $\alpha$ plays an important role in helping monocytes/macrophages penetrate inflammatory tissues, this chemokine is also expressed in human bone tissues of high bone remodeling ${ }^{12}$ and acts as a factor for inducing osteoclastogenesis in rat whole bone marrow cultures. ${ }^{11}$ Recently, it has been 
a
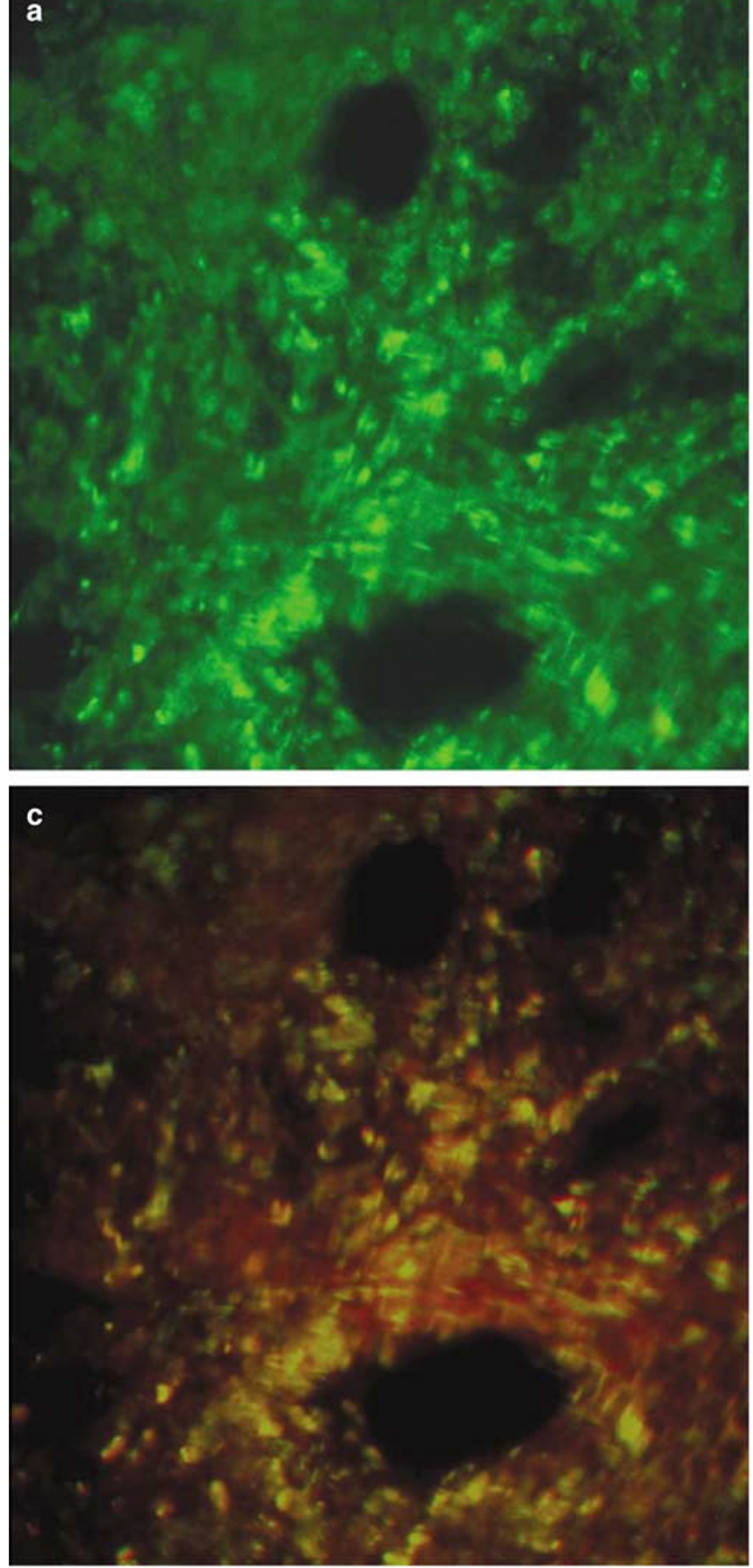

b

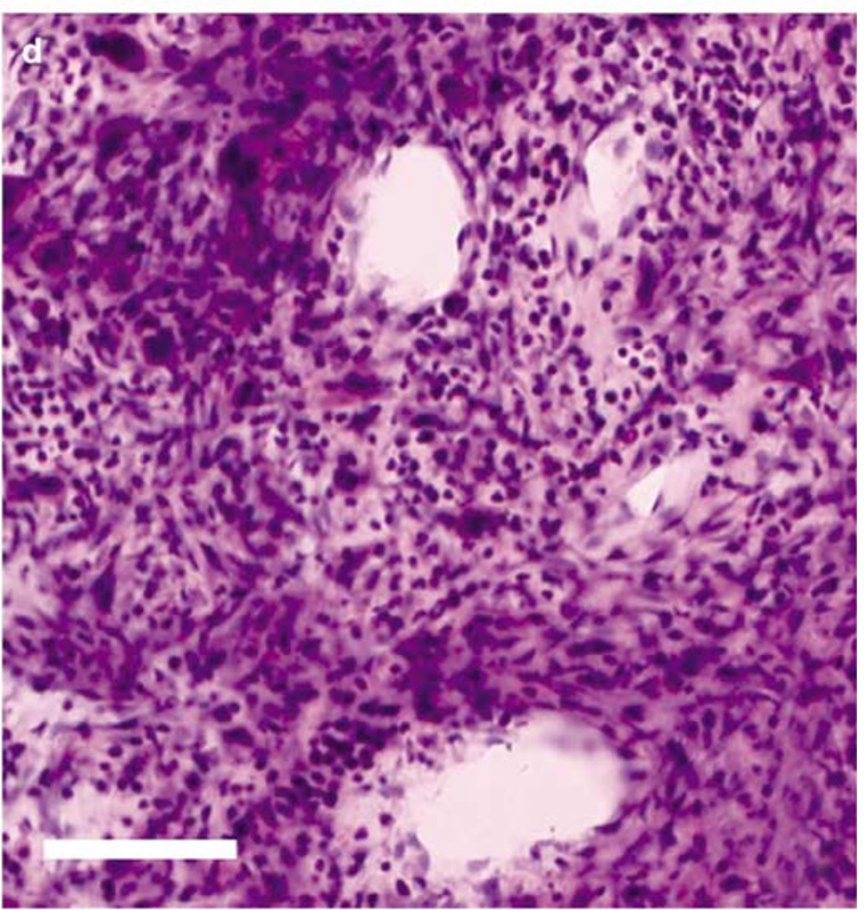

Figure 6 ED-1-positive cells produce MIP-1 $\alpha$. Photographs showing double-immunofluorescence staining of mononuclear cells in bone marrow cavity at 21 days after the adjuvant injection. (a) Localization of ED-1-antigen-positive cells (green). (b) Localization of MIP-1 $\alpha$ positive cells (red). (c) Merge of (a) and (b). Most of the ED-1-antigen-positive mononuclear cells expressed MIP-1 $\alpha$ (yellow). (d) Hematoxylin and eosin staining of bone marrow cavity in the distal end of tibia (an adjacent section with (a) and (b). Bar $70 \mu \mathrm{m}$.

revealed that MIP-1 $\alpha$ plays a key role in bone destruction accompanying multiple myeloma. ${ }^{13,14}$ A decrease in osteoclast progenitors in the diaphyseal portion of tibia might be related to an increase in the production of $\gamma$-interferon ( $\gamma$-IFN). Takayanagi et $a{ }^{18}$ showed a marked stimulation of osteoclastogenesis in $\gamma$-IFN-gene-deficient mice because of the absence of suppression of osteoclastogenesis by
$\gamma$-IFN. Suppression of osteoclastogenesis from marrow cells of diaphyseal tibia may be related to the recruitment of $\mathrm{T}$ lymphocytes expressing $\gamma$-IFN. Further studies are required regarding the production of $\gamma$-IFN in AA rats.

In our study, numerous cells secreting MIP- $1 \alpha$ were detected at the distal end of tibia of AA rats. We also confirmed that MIP- $1 \alpha$ had a significant 
chemotactic activity against normal bone marrow cells involving osteoclast progenitors. Most of the MIP-1 $\alpha$-producing cells were ED-1-positive macro-

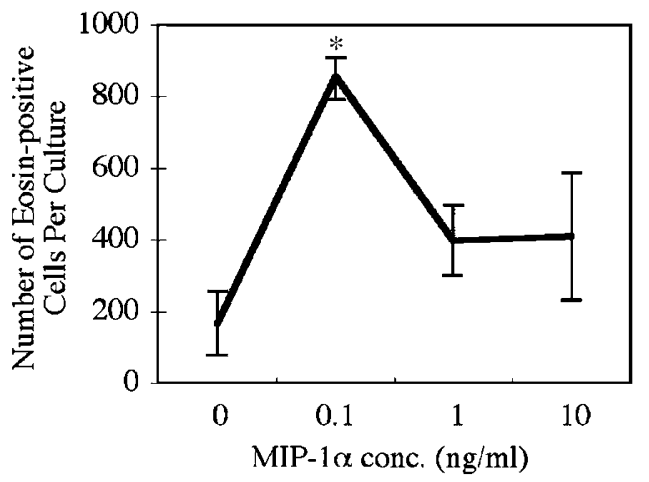

Figure 7 Chemotaxis of diaphyseal bone marrow cells toward MIP-1 $\alpha$. Rat bone marrow cells deprived of stromal cells were obtained as described in Materials and methods. To the upper side of the transwell, rat bone marrow cells deprived of stromal cells were added and to the lower parts of the transwell various concentrations of MIP- $1 \alpha$ were added. After $4 \mathrm{~h}$ of culture, cells that migrated to the lower side of the filter were fixed, stained, and counted under a microscope. Data were analyzed by Student's $t$-test. ${ }^{*} P<0.05$. A significant chemotactic activity was observed at $0.1 \mathrm{ng} / \mathrm{ml}$ of MIP-1 $\alpha$. Data represent a typical experiment from three independent experiments. phages. As few MIP- $1 \alpha$-producing macrophages were detected in normal bone marrow tissues, it is likely that activated macrophages induced by adjuvant injection commenced producing MIP- $1 \alpha$. Koch et a ${ }^{19}$ examined the level of MIP- $1 \alpha$ in the synovial fluid obtained from patients with RA and demonstrated a significant increase in the level of MIP- $1 \alpha$ in these patients in comparison to synovial fluid from osteoarthritis patients. They also compared MIP- $1 \alpha$ contents in peripheral blood and showed the up-regulation of MIP- $1 \alpha$ in patients with RA. It seems likely that MIP- $1 \alpha$ is produced by macrophages activated by inflammation. We have detected a small number of ED-1-positive macrophages that did not express MIP-1 $\alpha$. These cells are supposed to be MIP-1 $\alpha$-producing cells of nonmonocytes/macrophages lineage. It is interesting to investigate how these subpopulations change in relative ratio during the occurrence of arthritis. Hosaka et $a l^{20}$ and Lisignoli et $a l^{21}$ reported the presence of other chemokines, MCP-1, MIP-1 $\beta$, RANTES, IL-8, as well as MIP- $1 \alpha$ in the synovial tissues and in fluid obtained from patients with RA. Among these chemokines, MIP- $1 \alpha$ is the only one able to induce osteoclastogenesis. Our current findings strongly suggest a key role of MIP- $1 \alpha$ in the occurrence of severe bone destruction in AA
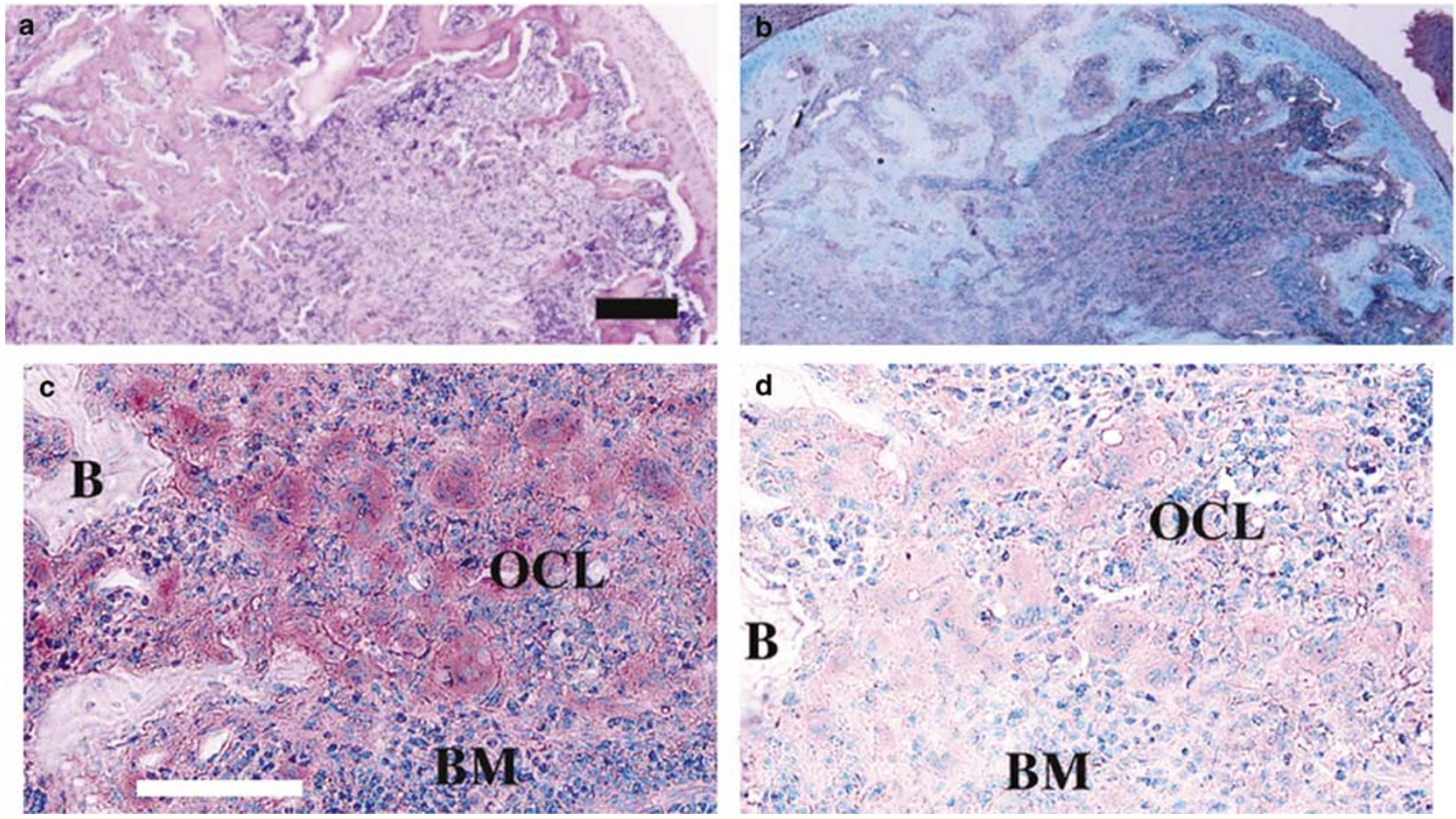

Figure 8 Marked expression of RANKL mRNA in aggregates of osteoclast-like cells in AA rats. Severe bone destruction was apparent in the distal end of tibia as well as in the adjacent tarsal bone in AA rats of 21 days after adjuvant injection. (a) Hematoxylin and eosin staining of the tarsal bone with severe bone destruction. (b-d) (adjacent section with (a): In situ hybridization (b) and (c), sections were hybridized with DIG-labeled anti-sense RNA probe for RANKL (c is the high magnification of b). (d) The serial section of (c) was hybridized with DIG-labeled sense RNA probe for RANKL. A significant expression of RANKL mRNA was apparent in osteoclast-like cells comprising aggregates of osteoclast-like giant cells in AA rats. OCL: Osteoclast-like multinucleated giant cells; BM: Bone marrow; B: Bone. (a, b) Bar $330 \mu \mathrm{m}$, (c, d) Bar $125 \mu \mathrm{m}$. 


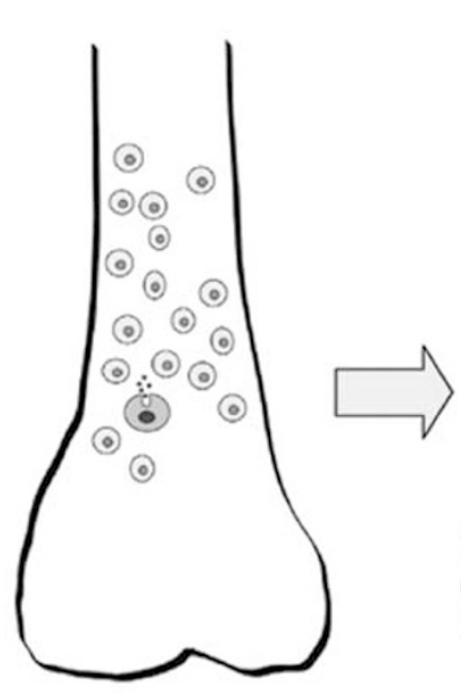

AA14days

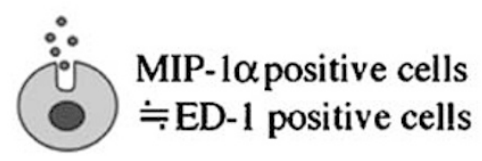

$?$

Osteoclast progenitors
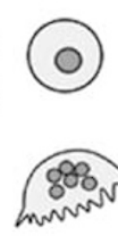

Osteoclast

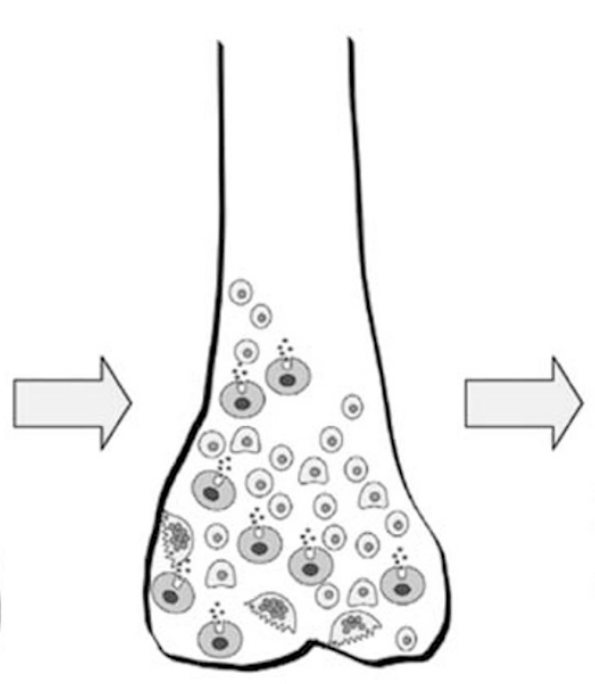

AA17days

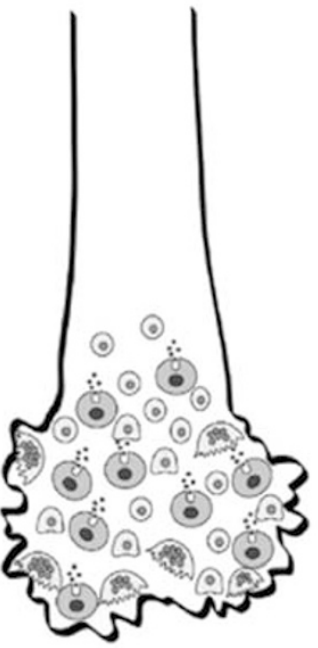

AA21days

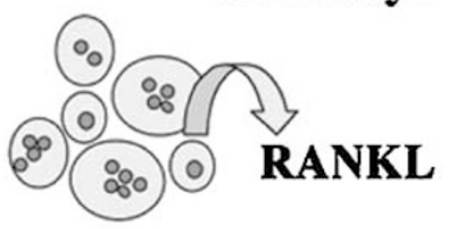

Aggregates of numerous Multinucleated osteoclast-like cells

Figure 9 Hypothesis-possible movement of osteoclast progenitors to the distal tibia by MIP-1 $\alpha$-mediated chemotaxis. In the early stages of adjuvant-induced arthritis before AA (17 days), MIP- $1 \alpha$-mediated migration of osteoclast progenitors to the distal tibia occurs. Migrated osteoclast progenitors are supposed to be fully differentiated to mature osteoclasts. Osteoclastogenesis as well as osteoclastic bone resorption in the distal tibia are enhanced by the presence of RANKL produced from the aggregates of osteoclast-like multinucleated giant cells present in the marrow cavity.

rats. A number of macrophages producing MIP-1 $\alpha$ were detected in the distal tibia of AA rats. Further detailed studies are required as to why MIP- $1 \alpha-$ producing macrophages accumulate in the distal tibia of AA rats. Elucidation of their homing mechanism seems to be important to regulate the severe bone destruction accompanying RA. The origin of these MIP- $1 \alpha$-producing macrophages needs to be clarified in the distal tibia. So far, it has not been reported whether these macrophages were moved from bone marrow tissue or if they came from synovial tissues.

Bone destruction in AA occurs by osteoclast bone resorption. Hirayama et $\mathrm{al}^{4}$ demonstrated augmentation in differentiation and activation of osteoclasts in patients with RA. RANKL is essential for normal osteoclastogenesis. This osteoclastogenic cytokine is also expressed in synovial cells ${ }^{22}$ and activated $\mathrm{T}$ cells ${ }^{23}$ in synovial tissues in patients with RA, which causes a marked stimulation of osteoclastogenesis in these patients. Kartsogiannis et $a l^{24}$ showed the expression of mRNA for RANKL in osteoclasts in normal bone by in situ hybridization. Roux et $a l^{25}$ demonstrated the expression of RANKL in osteoclast-like multinucleated cells of human giant cell tumor using an immunohistochemical technique. Our current study clearly showed expression of a high level of mRNA for RANKL in osteoclast-like multinucleated giant cells involved in the unique large-cell aggregates present in the bone marrow cavity accompanying severe bone destruction in AA rats. RANKL produced by these osteoclast-like multinucleated giant cells is supposed to relate closely to severe bone destruction in AA rats. Numerous MIP- $1 \alpha$-producing cells are detected in the distal tibia of AA rats. Recently, we have demonstrated that RANKL-dependent osteoclastogenesis is up-regulated by MIP- $1 \alpha{ }^{26}$ Abe et $a l^{27}$ reported that MIP- $1 \alpha$ is involved in bone destruction in patients with multiple myeloma. They showed that MIP- $1 \alpha$ stimulates osteoclastogenesis through RANKL production from stromal cells.

The current study demonstrated a possible migration of osteoclast progenitors from the diaphyseal marrow cavity to distal tibia in the early stage of AA rats, and that MIP- $1 \alpha$ is involved in these processes. Abnormal up-regulation of osteoclastogenesis is thought to occur in AA rats by the supply of abundant RANKL from aggregates of osteoclast-like giant cells and MIP- $1 \alpha$ secretion in the area of bone destruction. It should be possible to regulate bone destruction accompanying RA by controlling migra- 
tion and differentiation of osteoclast progenitors using a specific inhibitor for chemokine receptors as well as using specific inhibitors for RANKL signaling.

\section{Acknowledgements}

We thank Dr Dovie Wylie of On-site, English, Inc. (Palo Alto, CA, USA) for help with our English. We also thank Dr K Nagata and Dr Y Osaki of Kyushu University, Faculty of Dental Science for discussion. This work was partly supported by a Grant for Scientific Research from the Japanese Ministry of Education, Science and Culture (Project 14571737, 14571738).

\section{References}

1 Pearson CM, Wood FD. Studies of arthritis and other lesions induced in rats by the injection of mycobacterial adjuvant. VII. Pathologic details of the arthritis and spondylitis. Am J Pathol 1963;42:73-95.

2 Wu Z, Nagata K, Iijima T. Immunohistochemical study of NGF and its receptors in the synovial membrane of the ankle joint of adjuvant-induced arthritic rats. Histochem Cell Biol 2000;114:453-459.

3 Kuratani T, Nagata K, Kukita T, et al. Induction of abundant osteoclast-like multinucleated giant cells in adjuvant arthritic rats with accompanying disordered high bone turnover. Histol Histopathol 1998;13:751-759.

4 Hirayama T, Danks L, Sabokbar A, et al. Osteoclast formation and activity in the pathogenesis of osteoporosis in rheumatoid arthritis. Rheumatology 2002; 41:1232-1239.

5 Suda T, Nakamura I, Jimi E, et al. Regulation of osteoclast function. J Bone Miner Res 1997;12: 869-879.

6 Romas E, Gillespie MT, Martin TJ. Involvement of receptor activator of $\mathrm{NF}$ kappa $\mathrm{B}$ ligand and tumor necrosis factor-alpha in bone destruction in rheumatoid arthritis. Bone 2002;30:340-346.

7 Nakao M, Nomiyama H, Shimada K. Structures of human genes coding for cytokine LD78 and their expression. Mol Cell Biol 1990;10:3646-3658.

8 Menten P, Wuyts A, Damme JV. Macrophage inflammatory protein-1. Cytokine Growth Factor Rev 2002; 13:455-481.

9 Fuller K, Owens JM, Chambers TJ. Macrophage inflammatory protein-1 alpha and IL-8 stimulate the motility but suppress the resorption of isolated rat osteoclasts. J Immunol 1995;154:6065-6072.

10 Votta BJ, White JR, Dodds RA, et al. CKbeta-8 [CCL23], a novel CC chemokine, is chemotactic for human osteoclast precursors and is expressed in bone tissues. J Cell Physiol 2000;183:196-207.

11 Kukita T, Nakao J, Hamada F, et al. Recombinant LD78 protein, a member of the small cytokine family, enhances osteoclast differentiation in rat bone marrow culture system. Bone Miner 1992;19:215-223.

12 Kukita T, Nomiyama H, Ohmoto Y, et al. Macrophage inflammatory protein-1 alpha (LD78) expressed in human bone marrow: its role in regulation of hemato- poiesis and osteoclast recruitment. Lab Invest 1997;76:399-406.

13 Choi SJ, Cruz JC, Craig F, et al. Macrophage inflammatory protein 1-alpha is a potential osteoclast stimulatory factor in multiple myeloma. Blood 2000;96: 671-675.

14 Han JH, Choi SJ, Kurihara N, et al. Macrophage inflammatory protein-1 alpha is an osteoclastogenic factor in myeloma that is independent of receptor activator of nuclear factor kappa B ligand. Blood 2001; 97:3349-3353.

15 Kukita A, Kukita T, Hata K, et al. Heat-treated osteoblastic cell (ROS17/2.8)-conditioned medium induces the formation of osteoclast-like cells. Bone Miner 1993;23:113-127.

16 Kukita A, Kukita T, Shin JH, et al. Induction of mononuclear precursor cells with osteoclastic phenotypes in a rat bone marrow culture system depleted of stromal cells. Biochem Biophys Res Commun 1993; 196:1383-1389.

17 Kukita A, Kukita T, Ouchida M, et al. Osteoclastderived zinc finger (OCZF) protein with POZ domain, a possible transcriptional repressor, is involved in osteoclastogenesis. Blood 1999;94:1987-1997.

18 Takayanagi $\mathrm{H}$, Ogasawara K, Hida S, et al. T-cellmediated regulation of osteoclastogenesis by signalling cross-talk between RANKL and IFN-gamma. Nature 2000;408:600-605.

19 Koch AE, Kunkel SL, Harlow LA, et al. Macrophage inflammatory protein-1 alpha. A novel chemotactic cytokine for macrophages in rheumatoid arthritis. J Clin Invest 1994;93:921-928.

20 Hosaka S, Akahoshi T, Wada C, et al. Expression of the chemokine superfamily in rheumatoid arthritis. Clin Exp Immunol 1994;97:451-457.

21 Lisignoli G, Toneguzzi S, Pozzi C, et al. Chemokine expression by subchondral bone marrow stromal cells isolated from osteoarthritis (OA) and rheumatoid arthritis (RA) patients. Clin Exp Immunol 1999;116: 371-378.

22 Takayanagi $\mathrm{H}$, Iizuka $\mathrm{H}$, Juji $\mathrm{T}$, et al. Involvement of receptor activator of nuclear factor kappa B ligand/ osteoclast differentiation factor in osteoclastogenesis from synoviocytes in rheumatoid arthritis. Arthritis Rheum 2000;43:259-269.

23 Kong YY, Feige U, Sarosi I, et al. Activated T cells regulate bone loss and joint destruction in adjuvant arthritis through osteoprotegerin ligand. Nature 1999;402:304-309.

24 Kartsogiannis V, Zhou H, Horwood NJ, et al. Localization of RANKL (receptor activator of NF kappa B ligand) mRNA and protein in skeletal and extraskeletal tissues. Bone 1999;25:525-534.

25 Roux S, Amazit L, Meduri G, et al. RANK (receptor activator of nuclear factor kappa B) and RANK ligand are expressed in giant cell tumors of bone. Am J Clin Pathol 2002;117:210-216.

26 Watanabe T, Kukita T, Kukita A, et al. Direct stimulation of osteoclastogenesis by MIP-1 alpha: evidence obtained from studies using RAW 264 cell clone highly responsive to RANKL. J Endocrinol 2004; 180:193-201.

27 Abe M, Hiura K, Wilde J, et al. Role for macrophage inflammatory protein (MIP)-1 alpha and MIP-1 beta in the development of osteolytic lesions in multiple myeloma. Blood 2002;100:2195-2202. 